\title{
Lateral peritumoral lymphatic vessel invasion can predict lymph node metastasis in esophageal squamous cell carcinoma
}

\author{
Daisuke Mori ${ }^{1}$, Fumio Yamasaki ${ }^{1}$, Masami Shibaki ${ }^{1}$ and Osamu Tokunaga ${ }^{2}$ \\ ${ }^{1}$ Division of Pathology, Saga Prefectural Hospital Kouseikan, Saga, Japan and ${ }^{2}$ Faculty of Medicine, \\ Department of Pathology and Biodefense, Saga University, Saga, Japan
}

\begin{abstract}
Lymph node metastasis is an important prognostic factor in many types of cancer. We investigated the clinical significance of lymphangiogenesis and lymphatic vessel invasion in esophageal squamous cell carcinoma. We evaluated lymphatic vessel density and lymphatic vessel invasion in the intratumoral, peritumoral and normal compartments using D2-40 immunostaining. In addition, the peritumoral compartment was divided into the lateral peritumoral compartment and the non-lateral peritumoral compartment. The lymphatic vessel density was higher in the peritumoral and intratumoral compartments than in the normal compartment. However, the lymphatic vessel density did not correlate with any pathological parameters including lymph node metastasis. Intratumoral and peritumoral lymph vessels were small and collapsed while normal lymphatic vessels and lymphatic vessels with lymphatic vessel invasion were dilated and large. The presence of lymphatic vessel invasion, in the lateral peritumoral compartment but nowhere else, significantly correlated with lymph node metastasis. These results suggest that lymphangiogenesis might occur with esophageal cancer, but it does not play a direct role in lymphatic vessel invasion and lymph node metastasis. Peritumoral lymphatic vessel invasion, especially in the lateral peritumoral compartment, should imply a high probability of regional lymph node metastasis.
\end{abstract}

Modern Pathology (2007) 20, 694-700; doi:10.1038/modpathol.3800786; published online 27 April 2007

Keywords: esophageal squamous cell carcinoma; lymphangiogenesis; lymphatic vessel invasion; lateral peritumoral compartment; D2-40

Esophageal squamous cell carcinoma is one of the most common malignancies in the world. Its growth is relatively fast and it generally has a worse prognosis than any gastrointestinal tumor. Metastasis to regional lymph nodes represents the first step of dissemination in esophageal squamous cell carcinoma and serves as a major prognostic indicator for disease progression and as a guide for therapeutic decision- making. ${ }^{1}$

Previous studies have established the role of angiogenesis in tumor growth and hematogenous spread. However, despite the major role of the lymphatic vessels in the initial spread of cancer cells, little is known about the mechanisms whereby cancer cells enter the lymphatic system or whether nodal metastasis is dependent on tumor-induced

Correspondence: Dr D Mori, MD, Faculty of Medicine, Department of Pathology and Biodefense, Saga University, Nabeshima 5-1-1, Saga 849-8501, Japan.

E-mail: moridai@cc.saga-u.ac.jp

Received 02 January 2007; revised and accepted 20 February 2007; published online 27 April 2007 lymphangiogenesis or invasion of pre-existing lymphatic vessels. ${ }^{2}$ Until recently, the absence of a specific marker distinguishing lymphatic from blood vascular endothelia has been a significant hindrance to the study of the lymphatic system. In more recent years, monoclonal antibody D2-40 detects a fixation-resistant epitope on podoplanin. It was shown to be a selective marker for lymphatic endothelia, allowing the specific identification of lymphatic vessels in formalin- fixed, paraffinembedded tissues and the study of lymphatic vessel density in solid tumors. ${ }^{3-6}$

Lymphangiogenesis detected by antibodies specific for lymphatic endothelial cells has been associated with regional lymph node metastasis and a poor prognosis in head and neck squamous cell carcinoma, melanoma and cervical and pancreas cancer; however, recent studies on breast and prostate cancer seem to contradict this finding. ${ }^{5,7-11}$

The object of our study was to confirm the occurrence of lymphangiogenesis in esophageal squamous cell carcinoma by comparing the lymphatic vessel density in intratumoral, peritumoral 
and normal compartment; to verify the correlation of lymphatic vessel density and other pathological parameters; and to assess whether nodal metastasis is dependent on tumor-induced lymphangiogenesis or invasion of pre-existing lymphatic vessels.

\section{Materials and methods}

\section{Patients and Tumor Sampling}

The study included 46 consecutive patients with esophageal squamous cell carcinoma who were surgically treated at the Saga Prefectual Hospital and the Faculty of Medicine, Saga University. Because preoperative neoadjuvant therapy causes changes at the cancerous region, patients who had received preoperative chemotherapy or radiotherapy were excluded from this study. The definition of tumor stage and histological classification were made according to TMN classification criteria. ${ }^{12}$ Histological grades, tumor stages and depth of invasion are shown in Table 1. The cases of Tis (carcinoma in situ) were excluded for having too small an area to determine the lymphatic vessel density. Lymph node metastasis was present in 27 $(59 \%)$ cases, and the mean number of positive lymph nodes was 3.0 (range, 1-13).

\section{Immunohistochemistry}

The largest central sections of the entire tumor were reviewed with both hematoxylin-eosin and D2-40 immunohistochemical staining in each case. The mean number of sections per case was 3.6 (range, 2-6). Immunohistochemical assays were done on formalin-fixed, paraffin-embedded sections. Sections were cut and deparaffinized in xylene and rehydrated in graded alcohols. Antigen retrieval was done by heating slides for $40 \mathrm{~min}$ in citrate buffer $(\mathrm{pH} 6.0)$ at $95^{\circ} \mathrm{C}$. Endogeneous peroxidase activity was blocked by $3 \%$ hydrogen peroxide for $5 \mathrm{~min}$. Slides were incubated with the D2-40 monoclonal antibody (1:50 DakoCytomation, Glostrup, Denmark) for $30 \mathrm{~min}$ at room temperature. Immunohistochemical staining was performed on a DakoCytomation Autostainer using the EnVision + HRP DAB system (DakoCytomation, Glostrup, Denmark), according to the manufacturer's recommendations.

\section{Quantification of Lymphatic Vessels and Lymphatic Invasion}

The compartments were defined as follows. The intratumoral compartment was defined as the area located within the tumor mass. The peritumoral compartment was the 2-mm-wide area around the tumor mass. Moreover, the peritumoral compartment was divided into two compartments: The peritumoral compartment restricted at the mucosa and submucosa was defined as the lateral
Table 1 Relation between lymphatic vessel density and clinicopathological parameters in 46 esophageal squamous cell carcinoma

\begin{tabular}{|c|c|c|c|}
\hline Factor & Number of cases & $\begin{array}{l}\text { Intratumoral } \\
\quad( \pm \text { s.d. })\end{array}$ & $\begin{array}{l}\text { Peritumoral } \\
\quad( \pm \text { s.d. })\end{array}$ \\
\hline \multicolumn{4}{|l|}{ Age (years) } \\
\hline$<64.7$ & 25 & $26.8 \pm 8.3$ & $44.8 \pm 10.3$ \\
\hline$\geq 64.7$ & 21 & $26.4 \pm 11.3$ & $53.9 \pm 19.6$ \\
\hline \multicolumn{4}{|l|}{ Gender } \\
\hline Male & 38 & $27.3 \pm 10.4$ & $50.1 \pm 16.4$ \\
\hline Female & 8 & $22.9 \pm 6.4$ & $48.3 \pm 16.5$ \\
\hline \multicolumn{4}{|c|}{ Tumor size $(\mathrm{cm})$} \\
\hline$<4.7$ & 25 & $26.5 \pm 8.4$ & $47.1 \pm 15.5$ \\
\hline$\geq 4.7$ & 21 & $26.7 \pm 11.7$ & $52.9 \pm 17.5$ \\
\hline \multicolumn{4}{|c|}{ Depth of invasion } \\
\hline T1 & 15 & $27.1 \pm 8.2$ & $44.8 \pm 14.7$ \\
\hline $\mathrm{T} 2$ & 4 & $27.3 \pm 8.0$ & $58.5 \pm 19.4$ \\
\hline T3 & 27 & $26.2 \pm 11.3$ & $51.2 \pm 16.9$ \\
\hline \multicolumn{4}{|c|}{ Stage of disease } \\
\hline I & 8 & $25.4 \pm 9.0$ & $44.8 \pm 14.7$ \\
\hline II & 19 & $28.0 \pm 7.7$ & $53.1 \pm 18.2$ \\
\hline III & 19 & $25.7 \pm 12.4$ & $49.5 \pm 14.2$ \\
\hline \multicolumn{4}{|c|}{ Lymph node metastasis } \\
\hline Negative & 19 & $26.6 \pm 8.6$ & $49.0 \pm 19.1$ \\
\hline Positive & 27 & $26.6 \pm 11.0$ & $50.3 \pm 14.8$ \\
\hline \multicolumn{4}{|c|}{ Lymphatic vessl invasion } \\
\hline Negative & 22 & $25.5 \pm 10.0$ & $47.7 \pm 15.8$ \\
\hline Positive & 24 & $28.6 \pm 8.8$ & $53.0 \pm 17.7$ \\
\hline \multicolumn{4}{|c|}{ Venous invasion } \\
\hline Negative & 19 & $24.4 \pm 8.0$ & $42.4 \pm 17.2$ \\
\hline Positive & 27 & $28.1 \pm 11.0$ & $50.3 \pm 15.6$ \\
\hline \multicolumn{4}{|c|}{ Histological grade } \\
\hline G1 & 15 & $28.6 \pm 10.6$ & $53.9 \pm 18.7$ \\
\hline G2 & 29 & $26.2 \pm 9.6$ & $48.1 \pm 14.8$ \\
\hline G3 & 2 & $16.5 \pm 3.5$ & $42.0 \pm 28.2$ \\
\hline
\end{tabular}

G1, well differentiated; G2, moderately differentiated; G3, poorly differentiated; T1, tumor invades lamina propria or submucosa; T2, tumor invades muscularis propria; T3, tumor invades adventitia.

Classification was according to TMN criteria.

peritumoral compartment, and the remainder of the peritumoral compartment was the non-lateral peritumoral compartment (Figure 1). The area distant from the tumor was defined as the normal compartment. For D2-40 immunostaining, the slides were scanned at low magnification $(\times 4$ ocular and $\times 10$ objective), and the two highest density D2-40 positive vessels (hot spots) in the intratumoral, peritumoral and normal compartments were chosen. Lymphatic vessel density was determined by counting the number of D2-40 positive vessels in highpower magnification $(\times 20$ ocular and $\times 10$ object $)$ in the selected areas, then summing for a total vessels count. Lymphatic vessel invasion, defined as at least one cancer cell within D2-40 positive lymphatic vessels, was also recorded for each compartment. All slides were evaluated by two 


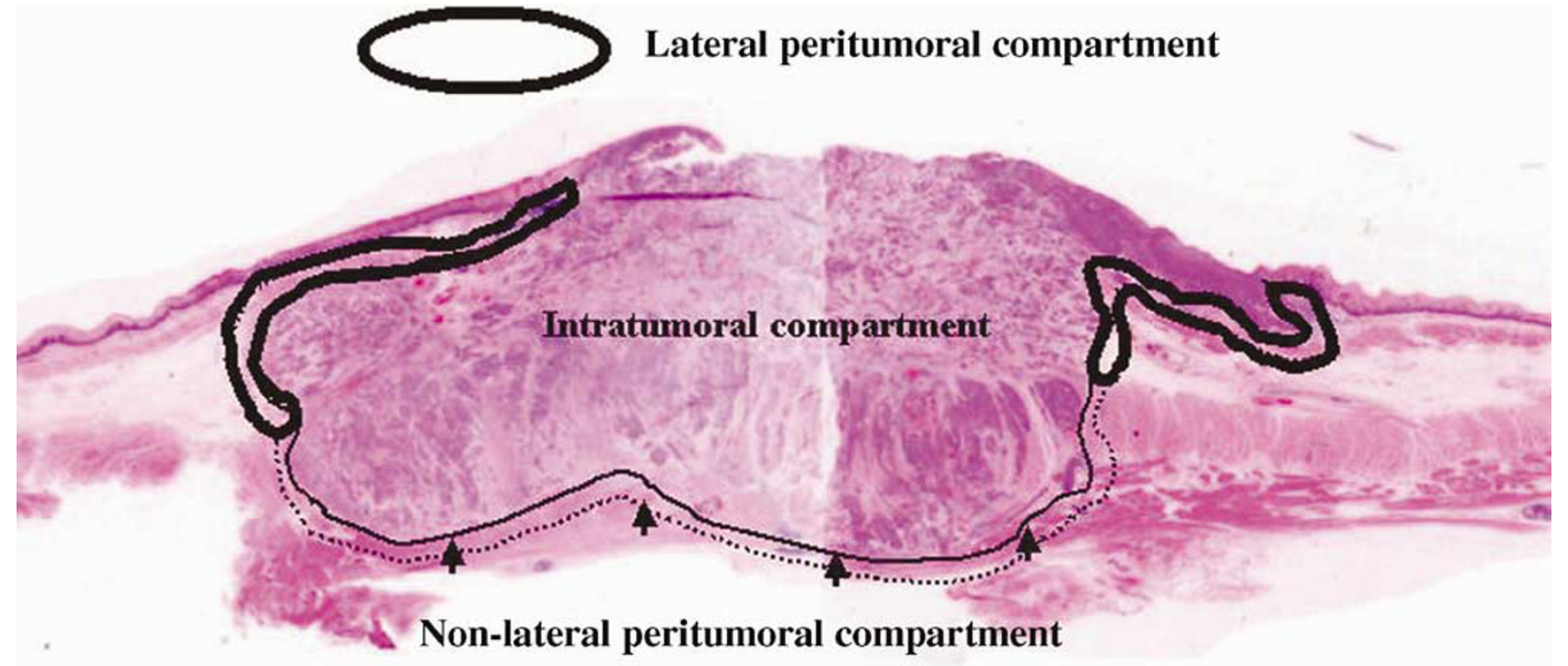

Figure 1 Schema of each compartment of esophageal squamous cell carcinoma.

investigators (DM and FY), and discrepancies were resolved by joint review of the slides through a multiheaded microscope. All cases were examined using an olympus DX51 microscope. The areas occupied by lymph vessels were calculated with a digital camera by filling system software (Flovel Co., Japan) in a $0.11 \mathrm{~mm}^{2}$ field of examination. ${ }^{13}$

\section{Statistical Analysis}

All statistical analyses were performed using the $\chi^{2}$-test when appropriate. Cox regression analysis was used for multivariate analysis, and an odds ratio with a $95 \%$ confidence interval was calculated for each factor. $P$-values $<0.05$ were considered statistically significant. All of the statistical tests were performed with StatView ver 5.0.

\section{Results}

Consistent with previous reports, this study showed that the antibody D2-40 was specific for lymphatic endothelial cells, and did not stain the vascular endothelial cells. ${ }^{4,5}$ The intratumoral, peritumoral and normal lymphatic vessel density were $26.6 \pm 18.1,49.7 \pm 20.4$ and $16.5 \pm 12.1$, respectively. Compared with normal lymphatic vessel density, peritumoral and intratumoral lymphatic vessel density were significantly increased. In addition, peritumoral lymphatic vessel density was significantly higher than intratumoral lymphatic vessel density in corresponding tumors (Table 2).

No lymphatic vessel density of any compartment correlated with any pathological parameter. Although peritumoral and intratumoral lymphatic vessel density in cases of positive lymph node
Table 2 Lymphatic vessel density at each compartment

\begin{tabular}{lcc}
\hline & Lymphatic vessel density & ( \pm s.d.) \\
\hline Intratumoral compartment & 26.6 & 18.1 \\
Peritumoral compartment & 49.7 & 20.4 \\
Normal compartment & 16.5 & 12.1
\end{tabular}

For all cases combined, $P<0.001$ for intratumoral vs peritumoral and intratumoral vs normal, and peritumoral vs normal.

metastasis was slightly higher than those of negative lymph node metastasis, no significant association was observed (Table 1).

Intratumoral and most peritumoral lymph vessels were small and collapsed (Figure 2a). In contrast, peritumoral lymph vessels were often enlarged and dilated in the mucosa and submucosa (Figure 2b). Most lymph vessels of the normal compartment were extended elliptically along the propria mucosa (Figure 2c). Most lymphatic vessels with lymphatic vessel invasion were much expanded, sometimes with tumor embolus (Figure 2d). The mean area of intratumoral, peritumoral and normal lymph vessels and lymphatic vessels with lymphatic vessel invasion were 526, 611, 3645 and $10977 \mu \mathrm{m}^{2}$ (Table 3).

Lymphatic vessel invasion was detected in 24 cases and the total number of invading lymphatic vessels was 175 . Lymphatic vessel invasion was present in a significantly higher percentage of cases with lymph node metastasis $(21 / 27,78 \%)$, as compared to those without lymph node metastasis $(3 / 19,16 \%)$. The presence of lymphatic vessel invasion correlated with lymph node metastasis (Table 4). Fifteen cases with lymphatic vessel invasion were present only in the peritumoral compartment, one case was only in the intratumoral 

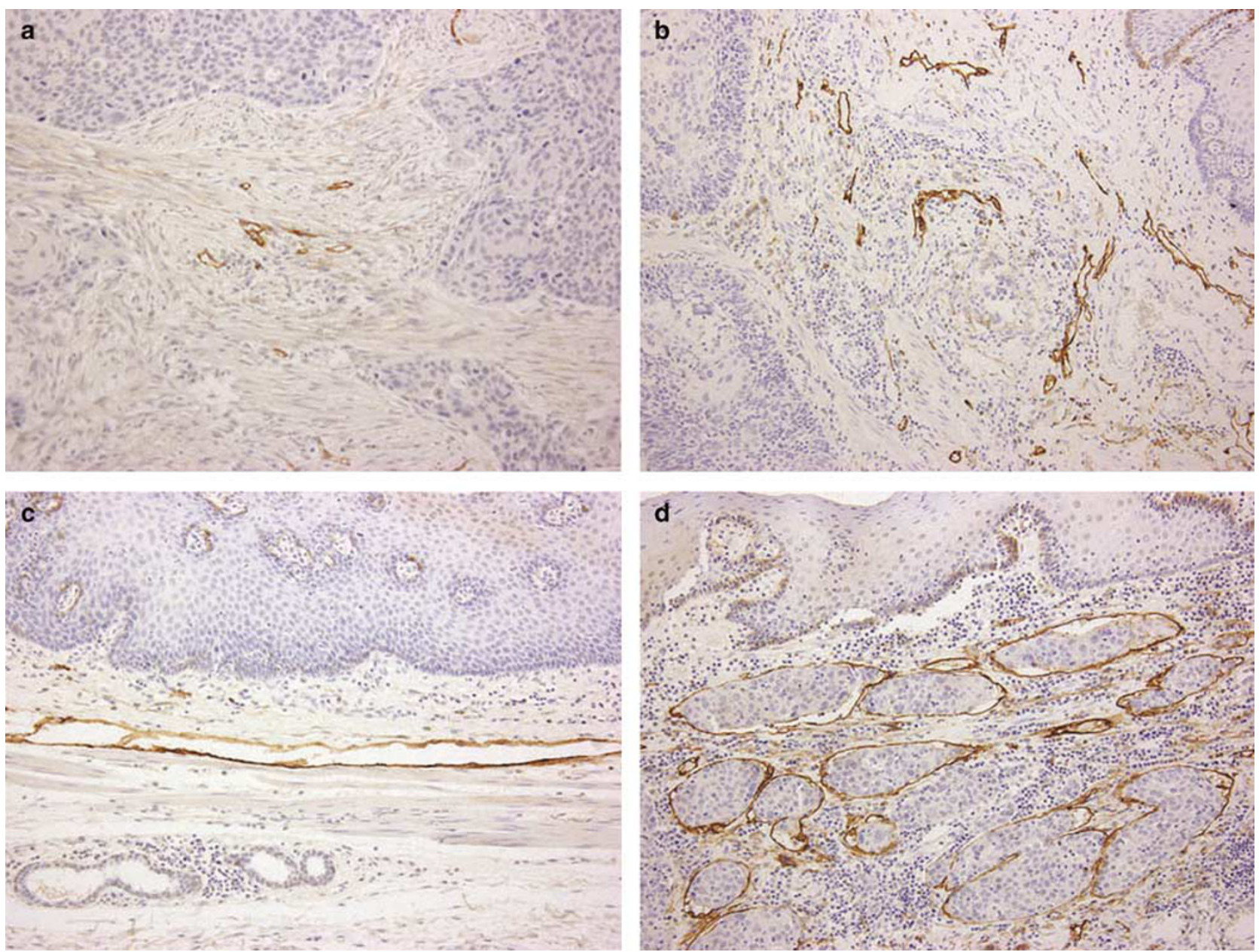

Figure 2 D2-40 stained lymphatic vessels of each compartment. Intratumoral lymph vessels were small and collapsed (a). Peritumoral lymph vessels were both small and collapsed and large and dilated (b). Lymph vessels of the normal compartment were extended elliptically along the propria mucosa (c). Tumor embolus that obliterated the lumen of lymphatic vessels was observed (d).

Table 3 Mean area of lymphatic vessel in each compartment

\begin{tabular}{lcc}
\hline & $\begin{array}{c}\text { Count of } \\
\text { lymphatic } \\
\text { vessel }\end{array}$ & $\begin{array}{c}\text { Mean area } \\
\text { of Lymphatic } \\
\text { vessel }\left(\mu m^{2}\right)\end{array}$ \\
\hline Intratumoral compartment & 383 & 526 \\
Peritumoral compartment & 535 & 611 \\
Normal compartment & 134 & 3645 \\
Lymphatic invasion & 175 & 10977 \\
\hline
\end{tabular}

compartment, and eight cases were with in both compartments. No lymphatic vessel invasion was identified in the normal compartment. Lymphatic vessel invasion was detected more in the peritumoral compartment, especially the lateral peritumoral compartment, than in the intratumoral compartment (Figure 3). Among all cases with lymphatic vessel invasion, lymphatic vessel invasion in the lateral peritumoral compartment occurred in 92\% (22/24). Moreover, among all invading lymphatic vessels, $81 \%(142 / 175)$ were located in the lateral peritu- moral compartment. The presence of lymphatic vessel invasion only in the lateral peritumoral compartment correlated with lymph node metastasis (Table 4).

\section{Discussion}

In this study, we have shown that (1) lymphatic vessel density is increased in the peritumoral and intratumoral compartment compared with the normal compartment; moreover, peritumoral lymphatic vessel density is significantly greater than intratumoral lymphatic vessel density; (2) the lymphatic vessel density does not correlate with any pathological parameters; (3) intratumoral and peritumoral lymphatic vessels are small and collapsed while normal lymphatic vessels and lymphatic vessels with lymphatic vessel invasion are dilated and large; and (4) the presence of lymphatic vessel invasion in the lateral peritumoral compartment, significantly correlates with lymph node metastasis. 
Table 4 Relation between lymphatic vessel invasion and lymph node metastasis in 46 cases

\begin{tabular}{|c|c|c|c|}
\hline & \multicolumn{2}{|c|}{ Lymphatic vessel invasion } & \multirow{2}{*}{$\begin{array}{l}\text { Sensitivity, } 78 \%(21 / 27) \\
\text { Specificity, } 84 \%(16 / 19) \\
\text { Accuracy, } 80 \%(37 / 46)\end{array}$} \\
\hline & + & - & \\
\hline LN meta (+) & 21 & 6 & \\
\hline LN meta $(-)$ & 3 & 16 & \\
\hline
\end{tabular}

LN meta: lymph node metastasis.

$P<0.0002$.

Relation between lymphatic vessel invasion in lateral peritumoral compartment and lymph node metastasis in 46 cases

\begin{tabular}{|c|c|c|c|}
\hline & $\begin{array}{l}\text { Lymphatic vessel invasion in } \\
\text { lateral peritumoral compartment }\end{array}$ & Otherwise & $\begin{array}{l}\text { Sensitivity, } 74 \%(20 / 27) \\
\text { Specificity, } 89 \%(17 / 19) \\
\text { Accuracy, } 80 \%(37 / 46)\end{array}$ \\
\hline LN meta (+) & 20 & 7 & \\
\hline LN meta $(-)$ & 2 & 17 & \\
\hline
\end{tabular}

Otherwise: lymphatic vessel invasion in non-lateral peritumoral compartment, intratumoral compartment and no lymphatic vessel invasion. $P<0.0001$.

Classically, it has been suggested that lymphatic vessels are located in the peritumoral area and such peritumoral lymphangiogenesis operates functionally in tumor development. ${ }^{14,15}$ Indeed, several reports have shown that increased peritumoral lymphatic vessel density is positively associated with lymph node metastasis and malignant aggressiveness in patients with head and neck squamous cell carcinoma, melanoma and cervical and pancreas cancer. ${ }^{5,7-9}$ However, contrary to these results, several other investigators reported that increased peritumoral lymphangiogenesis is associated with favorable biological behavior and good survival. ${ }^{16,17}$ Likewise, there exists a diversity of opinions as to the clinical significance of lymphangiogenesis. In this study, lymphangiogenesis actually occurred in esophageal squamous carcinoma because intratumoral and peritumoral lymphatic vessel density were greater than normal lymphatic vessel density. However, we did not find significant differences in the lymphatic vessel density between any pathological parameters including lymph node metastasis. The supposed reason was that peritumoral and intratumoral lymphatic vessels are predominantly small, collapsed and immature lymphatic vessels that cancer cells cannot enter. Actually, the mean area of peritumoral and intratumoral lymphatic vessels is much smaller than that of lymphatic vessel invasion. Moreover, we took into consideration that the number of lymphatic vessels is dependent on the states of the tumor surface. So the number of lymphatic vessels of a tumor with surface erosion or ulceration seemed to be increased..$^{18}$ There is evidence that inflammatory cells have an important role in pathological lymphangiogenesis. ${ }^{19}$

The presence of lymphatic vessel invasion in only the lateral peritumoral compartment correlated with lymph node metastasis, with a sensitivity of $74 \%$ (20/27). Lymphatic vessel invasion was most frequently found in the lateral peritumoral compartment $(81 \%)$. Lymphatic vessels of the lateral peritumoral compartment were intermingled small and collapsed, that is, lymphangiogenesis induced lymphatic vessels, and large and open, that is, preexisting lymphatic vessels. Lymphatic invasion in the lateral peritumoral compartment was predominantly observed in the large, open, preexisting lymphatic vessels, although this might be the influence of the tumor embolus. We hypothesized that lymphatic vessel invasion was frequently found in the lateral peritumoral compartment because preexisting lymphatic vessels mainly exist in the submucosal layer. These findings supported the hypothesis that peritumoral preexisting lymphatic vessels may be functionally important and are sufficient for lymphatic vessel invasion and lymph node metastasis. ${ }^{14}$ And Yonemura et $a l^{20}$ showed that in advanced gastric cancer, the main source of lymph node metastasis may be the lymphatic invasion in the submucosal layer.

Several reports have shown that lymphangiogenesis does not occur and active lymphangiogenesis does not play a role in lymphatic vessel invasion and lymph node metastasis in prostate cancer or breast cancer. And they proposed that the presence of pre-existing peritumoral lymphatic vessel invasion was more reliable at predicting lymph node metastasis. The presence of lymphangiogenesis might be different for different organs; however, pre-existing peritumoral lymphatic vessel invasion was most important for lymph node metastasis. ${ }^{11,17,21}$

In summary, lymphangiogenesis might be induced by esophageal carcinoma, but it does not play a direct role in lymphatic vessel invasion and lymph 


\section{References}
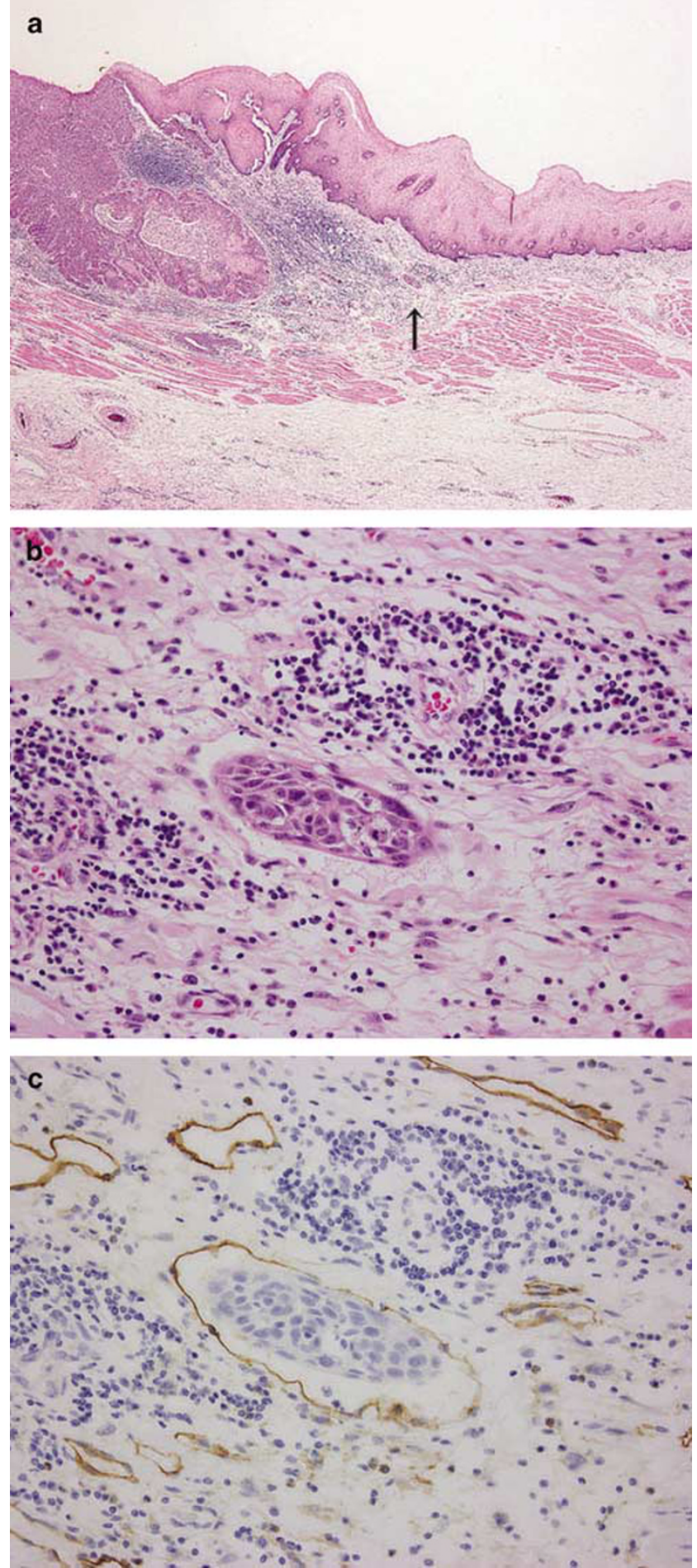

Figure 3 Lymphatic vessel invasions (arrow) were predominantly observed in the lateral peritumoral compartment at the same case of as Figure 1 (a) and high power magnification (b). A high power magnification cluster of cancer cells was detectd within the D2-40 positive lymphatic space (c).

node metastasis. Peritumor lymphatic vessel invasion, especially in the lateral peritumoral compartment, implies a high probability of regional lymph node metastasis.
1 Kitadai Y, Amioka T, Haruma K, et al. Clinicopathological significance of vascular endothelial growth factor (VEGF)-C in human esophageal squamous cell carcinomas. Int J Cancer 2001;93:662-666.

2 Stacker SA, Achen MG, Jussila L, et al. Lymphangiogenesis and cancer metastasis. Nat Rev Cancer 2002; 8:573-583.

3 Marks A, Sutherland DR, Bailey D, et al. Characterization and distribution of an oncofetal antigen (M2A antigen) expressed on testicular germ cell tumours. Br J Cancer 1999;80:569-578.

4 Kahn HJ, Marks A. A new monoclonal antibody, D2-40, for detection of lymphatic invasion in primary tumors. Lab Invest 2002;82:1255-1257.

5 Franchi A, Gallo O, Massi D, et al. Tumor lymphangiogenesis in head and neck squamous cell carcinoma: a morphometric study with clinical correlations. Cancer 2004;101:973-978.

6 Schacht V, Dadras SS, Johnson LA, et al. Up-regulation of the lymphatic marker podoplanin, a mucin-type transmembrane glycoprotein, in human squamous cell carcinomas and germ cell tumors. Am J Pathol 2005;166:913-921.

7 Dadras SS, Paul T, Bertoncini J, et al. Tumor lymphangiogenesis: a novel prognostic indicator for cutaneous melanoma metastasis and survival. Am J Pathol 2003; 162:1951-1960.

8 Hashimoto I, Kodama J, Seki N, et al. Vascular endothelial growth factor-C expression and its relationship to pelvic lymph node status in invasive cervical cancer. Br J Cancer 2001;85:93-97.

9 Rubbia-Brandt L, Terris B, Giostra E, et al. Lymphatic vessel density and vascular endothelial growth factor $C$ expression correlate with malignant behavior in human pancreatic endocrine tumors. Clin Cancer Res 2004;10:6919-6928.

10 Williams CSM, Leek RD, Robson AM, et al. Absence of lymphangiogenesis and intratumoral lymph vessels in human metastatic breast cancer. J Pathol 2003;200: 195-206.

11 Roma AA, Magi-Galluzzi C, Kral MA, et al. Peritumoral lymphatic invasion is associated with regional lymph node metastasis in prostate adenocarcinoma. Mod Pathol 2006;19:392-398.

12 Sobin LH, Wittekind C (eds). TMN Classification of Malignant Tumors, 6th edn. John Wiley: New York, 2003.

13 Auwera IV, Eynden GGV, Colpaert CG, et al. Tumor lymphangiogenesis in inflammatory breast carcinoma: a histomorphometric study. Clin Cancer Res 2005;11: 7637-7642.

14 Padera TP, Kadambi A, Tomaso E, et al. Lymphatic metastasis in the absence of functional intratumor lymphatics. Science 2002;296:1883-1885.

15 Isaka N, Padera TP, Hagendoorn J, et al. Peritumoral lymphatics induced by vascular endothelial growth factor-C exhibit abnormal function. Cancer Res 2004;64:4400-4444.

16 Straume O, Jackson DG, Akslen LA. Independent prognostic impact of lymphatic vessel density and presence of low-grade lymphangiogenesis in cutaneous melanoma. Clin Cancer Res 2003;9: 250-256.

17 Wong SY, Haack H, Crowley D, et al. Tumor-secreted vascular endothelial growth factor- $\mathrm{C}$ is necessary for 
prostate cancer lymphangiogenesis, but lymphangiogenesis in unnecessary for lymph node metastasis. Cancer Res 2005;65:9789-9798.

18 Schoppmann S. Lymphangiogenesis, inflammation and metastasis. Anticancer Res 2005;25:4503-4512.

19 Coussens LM, Werb Z. Inflammation and cancer. Nature 2002;420:860-867.
20 Yonemura Y, Endou Y, Tabachi K, et al. Evaluation of lymphatic invasion in primary gastric cancer by a new monoclonal antibody, D2-40. Hum Pathol 2006;37: 1193-1199.

21 Agarwal B, Saxena R, Moriyama A, et al. Lymphangiogenesis does not occur in breast cancer. Am J Pathol 2005;29:1449-1455. 